\title{
A Word from the Guest Editor
}

\section{Building Capacity for 21st Century Digital ELT Practices}

Communication in today's societies is increasingly digital. Twitter, texting, social networks, information and communication technologies (ICTs), augmented/virtual reality, and online gaming are transforming the way we use language, collaborate, and the way we teach and learn English (Chapelle \& Sauro, 2017; Kessler, 2018). A decade ago, we may have sent 65 texts a month to close friends; now, the average mobile phone user sends a minimum of 65 texts every 2 days (Van Camp, 2017). ICTs are inherently participatory and social, affording teachers an increasing array of synchronous and asynchronous forms of communication to explore genre, registers, and situationally specific interactions (Kessler et al., 2012). These emerging literacy practices involve new forms of social interaction, critical thinking, and language use along with new understandings of agency and community (Gee \& Hayes, 2011).

In addition, ICTs are disrupting the boundaries between time and space (Jones, 2016), offering distance, blended, flipped, and web-enhanced modalities that are transforming notions of the learning environment. Technology complexifies the relationship between a teacher and learners. Learners can now coproduce learning with technology alongside the instructor where teaching and learning roles are becoming ambiguous. Online teaching disrupts notions of social, teaching, and cognitive presence and requires the conscious curation of a "human feel" in online environments that can, at times, feel quite unfamiliar (Lawrence, 2014).

Web 2.0 tools such as wikis, Google docs, and social networks are intensely collaborative, transforming relationships between communicators and their interlocutors, between teachers and students (Davies, 2011; Luo, 2013). Corpora and digital databases of language use can build students' collocational competence, genre awareness, reading skills, and vocabulary (Boulton \& Cobb, 2017; Hadley \& Charles, 2017; Li, 2017). Learning management systems provide analytics tracking individual performance, enabling teachers to specifically target learner needs (Kessler, 2018; Stockwell, 2012). These technologies allow teachers to adopt innovative approaches to pedagogy: creating authentic, multimodal, action-oriented tasks; immersive experiences and individualized pathways to deepen language learning (Kessler, 2018); and a focus on developing langua-technoculture competence that Chapelle and Sauro (2017, p. 461) have highlighted as an ideal outcome of today's technology-mediated language learning landscapes: the intersection between technology, culture, and language that 21st century learners need to navigate on a daily basis. 


\section{Digital Expectations and Pressures}

As a result of this digital impact, English language teachers are often working with students who expect them to use technologies in ways that align with students' social and communicative practices (Kessler, 2018, p. 206). There is increasing pressure on teachers and stakeholders to integrate technology-mediated offerings into English language learning programs. As an administrator remarked in a feasibility study examining e-learning in Ontario adult English as a second language (ESL) non-credit programs, "E-learning is relevant because computer-based communication is needed for students' lives and they need computer skills to help them find jobs" (Lawrence, 2014, p. 131). There is a professional expectation that aspiring teachers will integrate technologies into their teaching (Kessler, 2018; Hubbard \& Kessler, 2017); many teachers are asked to demonstrate technology-integrated practices in hiring processes (Hubbard \& Kessler, 2017). TESOL International has published the TESOL Technology Standards (Healey et al., 2011) that establishes benchmarks for technology use by teachers and administrators.

In spite of these pressures, research continues to find that technology use in English language teaching is often constrained and not proportionate to the way technology is being used in everyday communication (Habbu, 2017; Hubbard \& Kessler, 2017). Inadequate tech access/support, time pressures, insufficient compensation, classroom-focused curricula combined with ineffective and often limited professional development often leave teachers unsupported in technology integration (Hubbard \& Kessler, 2017; Kessler, 2018; Tour, 2015). In addition, the exponential range of tech tools can be overwhelming. Even for early adopters, those teachers comfortable experimenting with new technologies, the choices today are daunting and can inhibit technology use.

A key challenge for teachers is not simply navigating the technologies but the methodological changes required to effectively use such tools (Dooly, 2009; Lawrence, 2018; Richards, 2015; Tour, 2015). Teaching with current technologies requires a paradigm shift in thinking about pedagogy and the learning environment. As Garrett (2009) has warned, we must not simply "computerize" what is already practiced in current classroom pedagogy. Instead, we must think of new ways of doing new things with these new tools. One of the big challenges with this is the visioning dilemma (Lawrence, 2018) where we teachers are unable to visualize how emerging technologies can potentially be used in language teaching practices without having seen them in use or having no personal experience with such technologies. Effective pedagogy that appropriately leverages technology-mediated practices requires an understanding of how to critically assess affordances and constraints of digital technologies to gauge their suitability for specific contexts (Haines, 2015). This is where theoretically informed research can help build a critical understanding of the affordances of technology-mediated 
practices. Such research can also offer insights into language learning processes and help build capacity for 21st century digital English language teaching practices.

The collection of articles in this Special Issue of the TESL Canada Journal examines a number of complementary areas to help inform teachers, teacher educators, researchers, and stakeholders trying to navigate the complexity of technology integration into language education. The first three full-length articles outline studies examining teacher capacity to use and integrate technology into language teaching practices. In "Digital Multimodal Composition and Second Language Teacher Knowledge," Schmidt reports on a case study investigating second language writing teachers' knowledge through the useful technological pedagogical content knowledge (TPACK) framework (Koehler \& Mishra, 2006). She examines how various knowledge areas support and/or constrain technology integration in postsecondary multimodal writing processes. Using an embedded case study approach, in "Language Teachers' Digital Mindsets: Links between Everyday Use and Professional Use of Technology," Cementina investigates the rarely examined personal versus professional beliefs and practices with technology use to identify how such beliefs/practices inform language teaching technology integration within a school community in the Philippines where educational resources are limited. This is followed by a discussion by Karamifar et al. on "Language Teachers and Their Trajectories Across Technology-Enhanced Language Teaching: Needs and Beliefs of ESL/EFL Teachers." They report on an international study examining ESL/EFL/second language teachers and teacher educator beliefs and needs around technology-focused professional development and perceived notions of the ideal language teacher and how these findings inform the need for continuing professional development.

The next two articles examine emerging approaches to technology use in English language teaching (ELT). In "The Multimodal Effects of Voice-Based Asynchronous Technology-Mediated Communication on EAP Speaking Performance," Sabbaghan et al. use a practitioner enquiry research method to investigate how a voice-based asynchronous technology-mediated framework can influence postsecondary English for Academic Purposes (EAP) learner fluency, accuracy, and intelligibility. In "Developing Cosmopolitan Communicative Competence Through Online Transnational Encounter," Wu and $\mathrm{Li}$ examine the potential of pedagogical activities within online transnational encounters between Hong Kong and U.S. university students to develop cosmopolitan communicative competence, a competence relevant to learners and educators in today's increasingly transnational communicative landscapes.

The sixth article in this issue, "Performance, Interaction, and Satisfaction of Graduate EAP Students in a Face-to-Face and an Online Class: A Comparative Analysis," by Stanchevici and Siczek adopts a comparative case study action research approach to investigate the different learning impacts of an 
online graduate-level English for Academic Purposes (EAP) course versus a face-to-face classroom delivery model, identifying the gaps and curricular needs in fully online EAP graduate courses. Recognizing the need for meaningful language use outside the classroom, Yeh and Swinehart (in "Social Media for Social Inclusion: Barriers to Participation in Target-Language Online Communities") examine learner perceptions of a social media platform to identify factors that inhibit full participation in online social networks among international postsecondary English language learners (ELL). The authors then use these findings to inform training strategies to help enhance ELL participation in such online communities.

In an In the Classroom piece titled "Creating Multimodal Reflections in an IEP Speaking-Listening Course," Macknish reports on strategies to encourage multimodal reflections using the example of university ELLs reflecting on a service-learning module to help students develop multiliteracies, autonomy, and learning strategies within an EAP listening-speaking course. In a Perspectives article, Lyddon outlines a five-step reflective approach using the Substitution-Augmentation-Modification-Redefinition (SAMR) model to help language educators make sound decisions around digital tool use. Lyddon's article is titled "Reflective Approach to Digital Technology Implementation in Language Teaching: Expanding Pedagogical Capacity by Rethinking Substitution, Augmentation, Modification, and Redefinition." The issue concludes with a book review by Roose discussing Zeng's book English Learning in the Digital Age: Agency, Technology, and Context, based on research examining Chinese English language learner perceptions of web-enhanced language learning for out-of-class learning. As Roose notes, this book sheds relevant insight on out-of-school language learning practices and valuable learning from student voices that can inform technology integration.

This collection of writing offers an exciting, multifaceted look at research and educational practices that can support capacity building for 21st century digital English language teaching and learning practices.

Geoff Lawrence (York University)

\section{Acknowledgements}

I'd like to express my gratitude to the TESL Canada Journal editors, Drs. Farahnaz Faez and Antonella Valeo, for including this special issue, and for their editorial assistant Michael Karas's guidance and support. I'd also like to extend a huge thanks to all authors and anonymous reviewers for their invaluable contributions, as well as to all those who submitted manuscripts for consideration. 


\section{References}

Boulton, A., \& Cobb, T. (2017). Corpus use in language learning: A meta-analysis. Language Learning, 67, 348-393.

Chapelle, C., \& Sauro, S. (Eds.). (2017). The handbook of technology and second language teaching. Hoboken, NJ: Wiley Blackwell.

Davies, R. J. (2011). Second-language acquisition and the information age: How social software has created a new mode of learning. TESL Canada Journal/Revue TESL du Canada, 28(2), 11-19.

Dooly, M. (2009). New competencies in a new era? Examining the impact of a teacher training project. ReCALL, 21(3), 352-369.

Garrett, N. (2009). Computer-assisted language learning trends and issues revisited: Integrating innovation. Modern Language Journal, 93, 719-740. https://doi.org/10.1111/j.15404781.2009.00969.x

Gee, J. P., \& Hayes, E. R. (2011). Language and learning in the digital age. London: Routledge.

Habbu, S. (2017). ESL teachers and their use of technology in the classroom: A replication study (Unpublished major research paper). York University, Toronto, ON, Canada.

Hadley, G., \& Charles, M. (2017). Enhancing extensive reading with data-driven learning. Language Learning \& Technology, 21, 131-152.

Haines, K. J. (2015). Learning to identify and actualize affordances in a new tool. Language Learning \& Technology, 19(1), 165-180.

Healey, D., Hanson-Smith, E., Hubbard, P., Ioannou-Georgious, S., Kessler, G., \& Ware, P. (2011). TESOL technology standards: Description, implementation, integration. Alexandria, VA: TESOL.

Hubbard, P., \& Kessler, G. (2017). Language teacher education and technology. In C. Chapelle \& S. Sauro (Eds.), The handbook of technology and second language teaching (pp. 277-291). Hoboken, NJ: Wiley Blackwell.

Jones, R. H. (2016). Digital literacies. In E. Hinkle (Ed.), Handbook of research into second language teaching and learning (Vol. 3; pp. 286-298). London: Routledge.

Kessler, G. (2018). Technology and the future of language teaching. Foreign Language Annals, 51, 205-218.

Kessler, G., Bikowski, D., \& Boggs, J. (2012). Collaborative writing among second language learners in academic web-based projects. Language Learning $\mathcal{E}$ Technology, 16, 91-109.

Kessler, G., \& Hubbard, P. (2017). Language teacher education and technology. In C. A. Chapelle, \& S. Sauro (Eds.), The handbook of technology and second language teaching and learning (pp. 278-292). Hoboken, NJ: Wiley-Blackwell.

Lawrence, G. (2014). A call for the human feel in today's increasingly blended world. In H. M. McGarrell \& D. Wood (Eds.), Contact Research Symposium, 40(2), 128-141.

Lawrence, G. (2018). The role of language teacher beliefs in an increasingly digitalized world. In B. Zou \& M. Thomas (Eds.), Integrating technology into contemporary language learning and teaching (pp. 140-160). Hershey, PA: IGI Global.

$\mathrm{Li}, \mathrm{S}$. (2017). Using corpora to develop learners' collocational competence. Language Learning $\mathcal{E}$ Technology, 21, 153-171.

Luo, T. (2013). Web 2.0 for language learning: Benefits and challenges for educators. International Journal of Computer-Assisted Language Learning and Teaching, 3(3), 1-17. https://doi.org/10.4018/ ijcallt.2013070101

Richards, J. C. (2015). The changing face of language learning: Learning beyond the classroom. RELC Journal, 46(1): 5-22.

Stockwell, G. (2012). Computer-assisted language learning: Diversity in research and practice. Cambridge, UK: Cambridge University Press.

Tour, E. (2015). Digital mindsets: Teachers' technology use in personal life and teaching. Language Learning \& Technology, 19(3), 124-139.

Van Camp, J. (2017). Tech is upending the ways we write, speak and even think. Digital Trends. Retrieved from https://www.digitaltrends.com/features/dt10-language-and-tech/ 


\section{Mot du rédacteur invité}

\section{Renforcement des capacités pour les pratiques numériques dans l'enseignement de l'anglais (ELT) au niveau avancé au $21^{\mathrm{e}}$ siècle}

La communication des sociétés actuelles passe de plus en plus par l'informatique. Twitter, les messages textes, les réseaux sociaux, les technologies de l'information et de la communication (TIC), la réalité augmentée ou virtuelle et les jeux en ligne sont autant de réalités qui transforment notre rapport au langage, nos façons de collaborer et nos manières d'enseigner et d'apprendre l'anglais (Chapelle \& Sauro, 2017; Kessler, 2018). Il y a dix ans, nous pouvions envoyer 65 textos par mois à nos amis proches alors que l'utilisateur de téléphone mobile moyen d'aujourd'hui en envoie au moins 65 tous les deux jours (Van Camp, 2017). Les TIC sont des réalités foncièrement participatives et sociales qui offrent aux enseignantes et aux enseignants une gamme grandissante de formes de communication synchrone et asynchrone leur permettant d'explorer des interactions spécifiques aux niveaux du genre, des registres et des situations (Kessler et al., 2012). Ces pratiques de littératie émergentes impliquent de nouveaux genres d'interaction sociale, de pensée critique et d'utilisation du langage ainsi que de nouvelles compréhensions des notions d'agentivité (capacité d'agir) et de communauté (Gee \& Hayes, 2011).

Les TIC perturbent également les frontières entre le temps et l'espace (Jones, 2016) en offrant des modalités à distance, mixtes, inversées et enrichies par le Web qui transforment les notions de l'environnement pédagogique. La technologie rend plus complexe la relation entre l'enseignement et l'apprentissage. Les apprenantes et apprenants peuvent désormais coréaliser leurs apprentissages avec leurs professeurs à l'aide de la technologie, ce qui brouille la distinction entre le rôle d'enseignant et celui d'apprenant. L'enseignement en ligne perturbe les notions de la présence sociale, pédagogique et cognitive en plus d'exiger l'établissement conscient dans les environnements en ligne d'un " visage humain » qui, par moments, peut créer un réel effet de dépaysement (Lawrence, 2014).

Intensément collaboratifs, des outils du Web 2.0 comme les wikis, Google Docs et les réseaux sociaux transforment les relations entre les communicateurs et leurs interlocuteurs, entre les professeurs et leurs élèves (Davies, 2011; Luo, 2013). Les corpus et bases de données numériques d'usages linguistiques peuvent aider les étudiantes et les étudiants dans les domaines des collocations, de la conscience du genre, des compétences en lecture et de l'acquisition de vocabulaire (Boulton \& Cobb, 2017; Hadley \& Charles, 2017; Li, 2017). Les systèmes de gestion de l'apprentissage en ligne fournis- 
sent des analyses de progrès individuels qui permettent aux professeurs de cibler les besoins particuliers des apprenantes et des apprenants (Kessler, 2018; Stockwell, 2012). Ces technologies permettent aux professeurs d'adopter des démarches pédagogiques novatrices : en créant des tâches authentiques, multimodales et axées sur l'action; des expériences immersives et des parcours individualisés qui approfondissent l'apprentissage linguistique (Kessler, 2018); et en mettant l'accent sur le développement d'une compétence "langua-technoculture » préconisée par Chapelle et Sauro (2017, p. 461) comme étant le prolongement idéal des paysages actuels de l'apprentissage linguistique soutenu par la technologie, c'est-à-dire $l^{\prime}$ intersection entre la technologie, la culture et la langue que les apprenantes et apprenants du $21^{\mathrm{e}}$ siècle doivent traverser au quotidien.

\section{Attentes et pressions numériques}

Cet impact numérique amène fréquemment les professeurs de langues à travailler avec des étudiantes et des étudiants qui s'attendent à ce que leur maniement des technologies soit à la hauteur de l'usage qu'elles ou ils en font personnellement dans leurs interactions sociales et leurs communications (Kessler, 2018, p. 206). Des pressions grandissantes sont exercées sur les enseignants et les intervenants pour les amener à intégrer la technologie aux programmes d'apprentissage de l'anglais. Comme l'observe un administrateur dans une étude de faisabilité portant sur les programmes hors compte d'enseignement de l'anglais langue seconde (ESL) aux adultes en Ontario, « l'apprentissage en ligne est pertinent parce que les apprenantes et les apprenants ont besoin de la communication automatisée dans leur vie et doivent posséder des compétences en informatique afin de pouvoir se trouver du travail » (Lawrence, 2014, p. 131). On s'attend, dans la profession, à ce que les futurs professeurs incorporent les technologies à leur enseignement (Kessler, 2018; Hubbard \& Kessler, 2017); plusieurs d'entre eux sont invités à démontrer leur maîtrise des pratiques d'intégration de la technologie au moment de leur embauche (Hubbard \& Kessler, 2017). TESOL International a pour sa part publié les TESOL Technology Standards (Healey et al., 2011) afin d'établir des points de référence pour l'utilisation de la technologie chez les professeurs et les administrateurs.

Malgré ces pressions, les recherches continuent de révéler que l'utilisation de la technologie au service de l'enseignement de l'anglais est fréquemment restreinte et qu'elle n'est pas proportionnelle au rôle de la technologie au service de la communication dans la vie de tous les jours (Habbu, 2017; Hubbard \& Kessler, 2017). Il arrive souvent que les professeurs soient laissés à eux-mêmes en matière d'intégration de la technologie en raison d'un manque d'accès à la technologie ou au soutien technique, de contraintes temporelles, de l'insuffisance de leur rémunération, de l'existence de cours centrés sur la salle de classe, le tout étant aggravé par des programmes de 
perfectionnement professionnel inefficaces et souvent limités (Hubbard \& Kessler, 2017; Kessler, 2018; Tour, 2015). Qui plus est, la portée exponentielle des outils technologiques peut être accablante, et la multiplicité des choix qui sont disponibles aujourd'hui peut être colossale et limiter l'utilisation de la technologie, et ce, même chez les professeurs qui l'ont adoptée au départ et se sont familiarisés avec l'expérimentation des nouvelles technologies.

Ce qui constitue un réel défi pour les professeurs au-delà de la maîtrise des technologies proprement dites, ce sont les modifications méthodologiques nécessaires pour l'utilisation efficace de tels outils (Dooly, 2009; Lawrence, 2018; Richards, 2015; Tour, 2015). L'enseignement à l'aide des technologies actuelles exige une transformation radicale de l'idée qu'on se faisait précédemment de la pédagogie et de l'environnement éducatif. Comme nous en avertissait Garrett (2009), nous ne pouvons pas nous contenter $\mathrm{d}^{\prime}$ « informatiser » les pratiques actuelles de la pédagogie en salle de classe. Nous devons plutôt concevoir de nouvelles façons de faire de nouvelles choses à l'aide de ces nouveaux outils. L'un des grands défis à surmonter à cet égard est le dilemme de la vision (Lawrence, 2018) qui nous empêche, nous les professeurs, de visualiser la façon dont les technologies émergentes pourraient éventuellement être incorporées aux pratiques de l'enseignement des langues sans les avoir déjà vues à l'œuvre ou en avoir nous-mêmes fait l'expérience. Pour pouvoir exercer une pédagogie qui sait mettre à profit des pratiques tributaires de la technologie, il faut d'abord s'adonner à une réflexion critique sur la façon de peser les affordances et les contraintes des technologies numériques de manière à pouvoir en évaluer la pertinence dans des contextes spécifiques (Haines, 2015). C'est à ce niveau-là que les recherches fondées sur la théorie peuvent éventuellement procurer une compréhension critique des affordances offertes par les pratiques pédagogiques soutenues par la technologie. De telles recherches peuvent également faire mieux comprendre les processus d'apprentissage linguistique et contribuer au renforcement des capacités pour les pratiques numériques liées à l'enseignement de l'anglais au $21^{\mathrm{e}}$ siècle.

Les articles sélectionnés pour le présent numéro spécial de la Revue TESL $d u$ Canada explorent un certain nombre de domaines complémentaires afin de mieux informer les professeurs, leurs formateurs, les chercheurs et les intervenants qui cherchent à s'y retrouver dans les dédales de l'intégration de la technologie à l'enseignement des langues. Les trois premiers articles de fond portent sur des études réalisées pour permettre de mieux comprendre la capacité des professeurs à utiliser et intégrer la technologie dans les pratiques de l'enseignement des langues. Dans l'article intitulé Digital Multimodal Composition and Second Language Teacher Knowledge, Schmidt décrit une étude de cas réalisée à l'aide du cadre TPACK (Technological Pedagogical Content Knowledge; Koehler \& Mishra, 2006). L'autrice examine la façon dont les différents domaines de connaissance soutiennent et/ou restreignent l'intégration de la technologie dans les processus de rédaction multimodaux au niveau post- 
secondaire. Dans l'article intitulé Language Teachers' Digital Mindsets: Links between Everyday Use and Professional Use of Technology, qui prend la forme d'une étude de cas intégrée, Cementina explore un domaine rarement abordé, celui de l'écart qui existe entre les croyances/pratiques personnelles et professionnelles relatives à l'utilisation de la technologie, afin de découvrir quelles sont les croyances/pratiques qui ont une influence sur l'intégration de la technologie à l'enseignement des langues à l'intérieur d'une communauté scolaire philippine à ressources éducatives limitées. Suit une discussion intitulée Language Teachers and Their Trajectories Across Technology-Enhanced Language Teaching: Needs and Beliefs of ESL/EFL Teachers dont les auteurs, Karamifar et al., traitent d'une étude internationale portant sur les croyances et les besoins des professeurs et des formateurs de professeurs d'anglais langue seconde (ESL)/langue étrangère (EFL) en mettant l'accent sur un perfectionnement professionnel axé sur la technologie et sur les idées qu'on se fait du professeur de langue idéal, et en analysant la façon dont ces constatations justifient le besoin d'un perfectionnement professionnel continu.

Les deux prochains textes examinent des approches émergentes face à l'utilisation de la technologie pour l'enseignement de l'anglais (ELT). Dans l'article intitulé The Multimodal Effects of Voice-Based Asynchronous Technology-Mediated Communication on EAP Speaking Performance, Sabbaghan et al. utilisent une méthode de recherche de type " enquête auprès des praticiens » pour voir dans quelle mesure un cadre asynchrone fondé sur la technologie et axé sur la voix peut influer sur la maîtrise linguistique, la précision et l'intelligibilité des apprenantes et apprenants de l'anglais académique (EAP) au niveau postsecondaire. Dans l'article intitulé Developing Cosmopolitan Communicative Competence Through Online Transnational Encounter, Wu et Li examinent la mesure dans laquelle les activités pédagogiques peuvent aider les étudiantes et les étudiants universitaires hongkongais et américains à développer une compétence communicative cosmopolite dans le cadre de leurs rencontres transnationales en ligne, compétence qui revêt grande importance tant pour les apprenants que pour les éducateurs dans les paysages de communication de plus en plus transnationaux d'aujourd'hui.

Rédigé par Stanchevici et Siczek, le sixième article du présent numéro, intitulé Performance, Interaction, and Satisfaction of Graduate EAP Students in a Face-to-Face and an Online Class: A Comparative Analysis, constitue une étude comparative de cas de type recherche-action permettant d'examiner les différents impacts pédagogiques d'un cours d'anglais académique (EAP) en les comparant au modèle de l'enseignement direct en classe et d'identifier les lacunes et les besoins des cours d'anglais académique de second cycle qui sont dispensés entièrement en ligne. Reconnaissant la nécessité d'utiliser judicieusement la langue anglaise en dehors de la salle de classe, Yeh et Swinehart (dans l'article intitulé Social Media for Social Inclusion: Barriers to Participation in Target-Language Online Communities) examinent les perceptions exprimées sur 
un média social par des apprenantes et des apprenants internationaux de la langue anglaise (ELL) au niveau postsecondaire afin d'identifier les facteurs qui les empêchent de participer pleinement à la vie des réseaux sociaux en ligne. Les auteurs se basent ensuite sur ces constatations pour proposer des stratégies de formation conçues pour accroître la participation de ces étudiantes et étudiants à l'intérieur de telles communautés en ligne.

Dans un article de la section En classe intitulé Creating Multimodal Reflections in an IEP Speaking-Listening Course, Macknish discute de stratégies aptes à encourager les réflexions multimodales des apprenantes et apprenants de l'anglais au niveau universitaire en donnant comme exemple les réflexions d'un groupe d'étudiantes et d'étudiants de niveau universitaire sur la pédagogie d'engagement communautaire (service-learning) concernant un module favorable au plurilinguisme, à l'autonomie et au développement de stratégies d'apprentissage à l'intérieur d'un cours intégré (écoute et parole) d'anglais à des fins universitaires (EAP). Dans un article de la section Perspectives, Lyddon décrit une approche réflective en cinq étapes à l'aide du modèle SAMR (Substitution, Augmentation, Modification, Redéfinition) conçue pour aider les professeurs de langues à prendre des décisions judicieuses concernant l'utilisation des outils numériques dans un article intitulé Reflective Approach to Digital Technology Implementation in Language Teaching: Expanding Pedagogical Capacity by Rethinking Substitution, Augmentation, Modification, and Redefinition. Ce numéro spécial se termine sur une recension signée Roose d'un livre de Zeng, English Learning in the Digital Age: Agency, Technology, and Context, basé sur des recherches portant sur les perceptions d'apprenantes et d'apprenants chinois de la langue anglaise relativement à l'enseignement enrichi par le Web en dehors de la salle de classe. Comme l'explique Roose, cet ouvrage offre un précieux éclairage sur les pratiques pédagogiques autres que scolaires et renferme des témoignages estudiantins qui peuvent être utiles pour l'intégration de la technologie.

Cette collection d'articles jette donc un regard passionnant et polyvalent sur les recherches et les pratiques éducatives qui peuvent contribuer au renforcement des pratiques numériques d'enseignement et d'apprentissage de l'anglais au $21^{\mathrm{e}}$ siècle.

Geoff Lawrence (Université York) 